\title{
Information-Theoretic Upper Bounds on the Capacity of Large Extended Ad Hoc Wireless Networks
}

\author{
Olivier Lévêque and İ. Emre Telatar, Member, IEEE
}

\begin{abstract}
We derive an information-theoretic upper bound on the rate per communication pair in a large ad hoc wireless network. We show that under minimal conditions on the attenuation due to the environment and for networks with a constant density of users, this rate tends to zero as the number of users gets large.
\end{abstract}

Index Terms-Ad hoc networks, capacity, cut-set bound, eigenvalues, random matrices.

\section{INTRODUCTION}

$\mathbf{T}$ HE feasability of large ad hoc wireless networks from an information-theoretic point of view is a subject of both mathematical and practical interest. An important issue is the evaluation of the capacity of such networks. In the seminal work of Gupta and Kumar [1], it has been shown that under some assumptions, the transport capacity of such (planar) networks scales like $\sqrt{n A}$ where $n$ is the number of users and $A$ is the area occupied by the network. The assumptions made in [1] state in particular that only point-to-point communications are allowed in the network and that interference is treated as noise. Even if these assumptions are quite realistic regarding state of the art wireless communications, the question remains whether the result obtained in there, more precisely the upper bound, can be confirmed from an information-theoretic point of view, that is, without any particular assumption on the way communications take place. A first confirmation of this result from an information-theoretic point of view has been obtained in [2]. It was, however, assumed in there that signals are strongly attenuated over distance (power decay of order $\frac{1}{r^{\alpha}}$ with $\alpha>6$ ).

The fact that the transport capacity scales with $\sqrt{n A}$ implies in particular that if there are order $n$ pairs in the network willing to establish communication at a common rate $R$ and if we assume that the pairs are chosen at random, without any consideration on the users' respective locations (so the average distance between paired users is of order $\sqrt{A}$ ), then the maximum achievable $R$ decreases like $\frac{1}{\sqrt{n}}$ as $n$ gets large. Our aim in the present paper is to give an information-theoretic proof of the fact that in this particular scenario (and for a uniformly distributed network with a constant density of users), the maximum achievable $R$ tends to zero under a minimal assumption on the attenuation function (power decay of order $\frac{1}{r^{\alpha}}$ with $\alpha>2$ ).

Manuscript received August 20, 2003; revised September 14, 2004. This work was supported in part by the National Competence Center in Research on Mobile Information and Communication Systems (NCCR-MICS), a center supported by the Swiss National Science Foundation under Grant 5005-67322.

The authors are with the Ecole Polytechnique Féderale de Lausanne, IC-ISC-LTHI, Bâtiment INR, Station 14, CH-1015 Lausanne, Switzerland (e-mail: olivier.leveque@epfl.ch; emre.telatar@epfl.ch).

Communicated by A. Lapidoth, Associate Editor for Shannon Theory.

Digital Object Identifier 10.1109/TIT.2004.842576
We would like to point out that our result does not say anything about the transport capacity of the network in general. Moreover, our upper bound is not as tight as the $\frac{1}{\sqrt{n}}$ behavior found in [1], [2] under stronger assumptions.

Our results apply to $d$-dimensional networks (see also [3] for an extension of the results of [1] to three dimensions). We also consider the model where an additional exponential factor is present in the attenuation function (as also considered in [2]). Section II is devoted to the study of uniformly distributed networks. In Section III, we consider the particular situation of a "regular" network, where the users are placed on a grid.

\section{UNIFORMLY DisTRIBUTED NETWORKS}

The network we consider consists of an even number $n$ of users independently and uniformly distributed in the $d$-dimensional region

$$
\Omega_{n}=\left[-n^{1 / d}, n^{1 / d}\right] \times\left[0, n^{1 / d}\right]^{d-1}
$$

of volume $\left|\Omega_{n}\right|=2 n$, therefore, expanding with the number of users (when $d=1, \Omega_{n}$ is the interval $[-n, n]$ and when $d=2$, $\Omega_{n}$ is the rectangle $\left.[-\sqrt{n}, \sqrt{n}] \times[0, \sqrt{n}]\right) .{ }^{1}$ Note that because of this assumption of an "extended" network, the density of users remains constant as $n$ increases.

Let us divide these users into two arbitrary groups of $n / 2$ users and assume that each user of the first group wishes to establish (one-way) communication with a correspondent chosen at random in the second group (without any consideration on their respective locations). ${ }^{2}$ We assume that there is no fixed infrastructure that helps relaying communications, but we also assume no restriction on the kind of help the users can give to each other; in particular, any user may act as a relay for the communicating pairs. We further assume that in order to establish communication, each user has a device of power $P$. The attenuation of the transmitted signals over distance is governed by the function $g(r)$ given by ${ }^{3}$

$$
g(r)=\frac{e^{-\beta r / 2}}{r^{\alpha / 2}}, \quad r>0 .
$$

\footnotetext{
${ }^{1}$ The fact that the region $\Omega_{n}$ is rectangular, and not square, is of little importance since we are only interested in the asymptotic behavior of the network capacity.

${ }^{2}$ It could be raised here that this situation does not take place in a real network; however, the argument developed hereafter holds even if only a constant fraction of the users wish to establish communication without any consideration on their respective locations.

${ }^{3}$ One could raise again an objection here: without any constraint on the minimum distance between users, the above attenuation function may take arbitrarily large values. Because of our assumption of "extended" network, however, points are likely to be sufficiently far apart form each other.
} 
Note that $g$ describes the decay of the amplitude of the electric field and not that of the power. This model of decay is accepted as a standard one in wireless communications. The case $\alpha=2$ and $\beta=0$ describes the decrease of the electric field in empty space. Because of canceling reflections, the coefficient $\alpha$ is usually taken to be greater than 2 for terrestrial transmissions, whereas a nonzero exponential factor $\beta$ takes into account absorption in the air.

Let now $R$ be the maximum achievable rate per communication pair in the network. We prove in the following that $R$ tends almost surely to zero as $n$ gets large, under the assumption that either $\alpha>d \vee 2(d-2)=\max (d, 2(d-2))$ or $\beta>0$. In the particular case $d=2$, our result says more precisely that

$$
R \leq K \frac{n^{1 / \alpha} \log n}{\sqrt{n}}
$$

when $\alpha>2$ and $\beta=0$, and that

$$
R \leq K \frac{(\log n)^{2}}{\sqrt{n}}
$$

when $\beta>0$ (see Theorems 2.5 and 2.10). We therefore see that in the case $\beta=0$, the bound obtained is quite distant from the $\frac{1}{\sqrt{n}}$ bound of [1], especially when $\alpha$ is small.

As a first step, we divide the domain $\Omega_{n}$ into two equal parts separated by the hyperplane $x^{(1)}=0$, where $x^{(i)}$ denotes the $i$ th coordinate of $x \in \Omega_{n}$. Statistically, there are about $n / 2$ users on the left-hand side of the domain; moreover, about half of these are transmitters and half of these transmitters wish to establish communication with a receiver on the right-hand side of the domain. In total, there are therefore about $n / 8$ communications which need to cross the imaginary boundary from left to right, and it is easy to see that as $n$ gets large, deviations from the average are of order much smaller than $n$ with high probability.

In order to obtain an upper bound on $R$, we make a series of optimistic assumptions: we first assume that only the above $n / 8+o(n)$ communications need to be established. We then introduce $n$ additional "mirror" users that help relaying communications (where the mirror location of $x \in \Omega_{n}$ is $\left.\tilde{x}=\left(-x^{(1)}, x^{(2)}, \ldots, x^{(n)}\right)\right)$. We see that there are now exactly $n$ users on each side of the domain, which are moreover independently and uniformly distributed on each side. There is, however, a more important reason for introducing these "mirror" users: it brings a helpful symmetry in the problem, as we shall see later (Remark 2.1). On the other hand, doubling the number of users has no influence on the asymptotic behavior of the capacity.

Let us further assume that all the users on the left-hand side can share instantaneous information and even distribute their power resources among themselves in order to establish communication in the most efficient way with the users on the righthand side, which in turn are able to distribute the received information instantaneously among themselves. We also assume that the user locations are known to all users. Following the argument of [4, Theorem 14.10.1], we obtain the following upper bound on the sum of the rates of communications going from left to right:

$$
\sum_{x \in S, y \in S^{c}} R_{x y} \leq I\left(X_{S} ; Y_{S^{c}} \mid X_{S^{c}}\right)
$$

where $S$ denotes the set of users' locations on the left-hand side of $\Omega_{n}$ and $S^{c}$ those on the right-hand side; $X_{S}=\left(X_{x}, x \in S\right)$ denotes the messages sent by the users in $S, Y_{S^{c}}$ those received by the users in $S^{c}$, and $X_{S^{c}}$ those sent back by the users in $S^{c}$ (which takes into account the effect induced by some eventual feedback).

In our setting, we have the following formal relation between $X_{S}$ and $Y_{S^{c}}$ :

$$
Y_{S^{c}}=G X_{S}+Z
$$

where $G=\left(G_{x y}, x \in S, y \in S^{c}\right)$ is the matrix whose entries are given by $G_{x y}=g(|x-y|)$, with $g$ given by (1), and $Z$ is independent additive white Gaussian noise. From this relation, we deduce that

$$
\begin{aligned}
I\left(X_{S} ; Y_{S^{c}} \mid X_{S^{c}}\right)= & H\left(G X_{S}+Z \mid X_{S^{c}}\right) \\
& -H\left(G X_{S}+Z \mid X_{S}, X_{S^{c}}\right) \\
= & H\left(G X_{S}+Z \mid X_{S^{c}}\right)-H(Z) \\
\leq & H\left(G X_{S}+Z\right)-H(Z) \\
= & H\left(G X_{S}+Z\right)-H\left(G X_{S}+Z \mid X_{S}\right) \\
= & I\left(X_{S} ; Y_{S^{c}}\right)
\end{aligned}
$$

where we have used the fact that $Z$ is independent from the other variables and that conditioning reduces entropy.

From now on, we will adopt the following notations (since we know that there are exactly $n$ users on each side):

$$
\begin{array}{rlrl}
S & =\left\{x_{1}, \ldots, x_{n}\right\}, \quad S^{c} & =\left\{y_{1}, \ldots, y_{n}\right\} \\
X_{S} & =\left(X_{1}, \ldots, X_{n}\right), & Y_{S^{c}} & =\left(Y_{1}, \ldots, Y_{n}\right)
\end{array}
$$

where we assume that $\left(x_{i}, y_{i}\right)$ forms a pair of "mirror" users for each $i \in\{1, \ldots, n\}$. With this notation, the channel model (2) becomes

$$
Y_{j}=\sum_{i=1}^{n} G_{i j} X_{i}+Z_{j}, \quad j=1, \ldots, n
$$

where $G_{i j}=g\left(\left|x_{i}-y_{j}\right|\right)$ and $Z=\left(Z_{1}, \ldots, Z_{n}\right)$ is a vector of independent circularly symmetric complex Gaussian random variables with unit variance. Under the power constraint

$$
\sum_{i=1}^{n} \mathbb{E}\left(\left|X_{i}\right|^{2}\right) \leq n P
$$

(arising from the fact that the users are assumed to be able to distribute their power resources among themselves), the mutual information $I\left(X_{1}, \ldots, X_{n} ; Y_{1}, \ldots, Y_{n}\right)$ is maximum when $\left(X_{1}, \ldots, X_{n}\right)$ is a jointly Gaussian vector with some covariance matrix $Q$, so

$$
\begin{aligned}
\sum_{i, j=1}^{n} R_{i j} & \leq \max _{p_{X}: \sum_{i} \mathbb{E}\left(\left|X_{i}\right|^{2}\right) \leq n P} I\left(X_{1}, \ldots, X_{n} ; Y_{1}, \ldots, Y_{n}\right) \\
& =\max _{Q \geq 0: \operatorname{Tr}(Q) \leq n P} \log \operatorname{det}\left(I+G Q G^{\dagger}\right) .
\end{aligned}
$$

By unitary transformation of the matrix $G$ (see, e.g., [5]), one finally obtains

$$
\sum_{i, j=1}^{n} R_{i j} \leq \max _{P_{k} \geq 0: \sum_{k=1}^{n} P_{k} \leq n P} \sum_{k=1}^{n} \log \left(1+P_{k} \lambda_{k}^{2}\right)=: C_{n}
$$


where $\lambda_{k}$ are the singular values of the matrix $G$ (in decreasing order and repeated by multiplicity). Let us recall that about $n / 8$ communications need to be established from left to right and that we wish to achieve the same common rate $R$ for all these communications. In order to prove that $R$ tends to zero as $n$ gets large, it is therefore sufficient to prove that the capacity $C_{n}$ defined in (3) grows sublinearly in $n$, that is, decreases to zero when divided by $n$.

Note finally that $y_{i}^{(1)}=-x_{i}^{(1)}$ and $y_{i}^{(k)}=x_{i}^{(k)}$ for $k \in$ $\{2, \ldots, d\}$, so the matrix $G$ is symmetric; it has therefore real eigenvalues $\mu_{k}$ and the singular values $\lambda_{k}$ are equal to $\left|\mu_{k}\right|$.

\section{A. No Absorption Case}

In this subsection, we assume that $\beta=0$, i.e., there is no absorption which creates an exponential decay of the power over the distance. It is shown in the Appendix that if

$$
g(r)=\frac{1}{r^{\alpha / 2}}, \quad r>0
$$

and $\alpha>2(d-2) \vee 0$, then $G$ is a nonnegative definite matrix, so its eigenvalues $\mu_{k}$ are also nonnegative (and equal to $\lambda_{k}$ ).

We can, therefore, obtain the following successive upper bounds on $C_{n}$. Noting first that $P_{k} \leq n P$ for all $k$, we obtain

$$
C_{n} \leq \sum_{k=1}^{n} \log \left(1+n P \mu_{k}^{2}\right) .
$$

Since $\mu_{k} \geq 0$, we further obtain

$$
\begin{aligned}
C_{n} & \leq \sum_{k=1}^{n} \log \left(\left(1+\sqrt{n P} \mu_{k}\right)^{2}\right) \\
& =2 \sum_{k=1}^{n} \log \left(1+\sqrt{n P} \mu_{k}\right) .
\end{aligned}
$$

The computation of the asymptotic behavior of the eigenvalues $\mu_{k}$ is not an easy task. We are therefore going to use the following majorization argument: from [6, p. 218, Theorem 9.B.1], we know that the eigenvalues $\mu_{k}$ majorize the diagonal elements of $G$, that is,

$$
\sum_{k=1}^{l} \mu_{k} \geq \sum_{k=1}^{l} G_{k k}, \quad \forall l \in\{1, \ldots, n-1\}
$$

and

$$
\sum_{k=1}^{n} \mu_{k}=\sum_{k=1}^{n} G_{k k}
$$

On the other hand, by [6, p. 64, Proposition 3.C.1], we know that the function

$$
\left(x_{1}, \ldots, x_{n}\right) \mapsto \sum_{k=1}^{n} \log \left(1+\sqrt{n P} x_{k}\right)
$$

is Schur-concave (recall [6, p. 54, Definition 3.A.1]: a Schur-concave funtion is a function $f: \mathbb{R}^{n} \rightarrow \mathbb{R}$ such that $f\left(x_{1}, \ldots, x_{n}\right) \leq f\left(y_{1}, \ldots, y_{n}\right)$ as long as $\left(x_{1}, \ldots, x_{n}\right)$ majorizes $\left(y_{1}, \ldots, y_{n}\right)$ in the sense defined above). We therefore conclude that

$$
C_{n} \leq 2 \sum_{k=1}^{n} \log \left(1+\sqrt{n P} G_{k k}\right) .
$$

Moreover, $G_{k k}=g\left(\left|x_{k}-y_{k}\right|\right)=g\left(2\left|x_{k}^{(1)}\right|\right)$, so

$$
C_{n} \leq D_{n}^{0}:=2 \sum_{k=1}^{n} \log \left(1+\sqrt{n P} g\left(2\left|x_{k}^{(1)}\right|\right)\right) .
$$

Remark 2.1: Note that the preceding majorization argument does not work in general if we replace the eigenvalues $\mu_{k}$ by the singular values $\lambda_{k}$ : this is because the singular values of the matrix $G$ satisfy (4) but not (5). This explains why we need $G$ to be nonnegative definite, and motivate the introduction of mirror users.

In order to obtain an upper bound on the average behavior of $D_{n}^{0}$, we need the following technical lemma.

Lemma 2.2: For any $C, p>0$ and $\alpha>1$, there exists a constant $K>0$ such that for all sufficiently large $n$, we have

$$
\int_{0}^{n} d x \log \left(1+\frac{C n^{p}}{x^{\alpha}}\right) \leq K n^{\frac{p}{\alpha} \wedge 1} \log n
$$

where $a \wedge b$ denotes the minimum value of $a$ and $b$.

Proof: Let us define $x_{0}=n^{\frac{p}{\alpha} \wedge 1}$ and compute

$$
\begin{array}{rl}
\int_{0}^{n} & d x \log \left(1+\frac{C n^{p}}{x^{\alpha}}\right) \\
= & \int_{0}^{1} d x \log \left(1+\frac{C n^{p}}{x^{\alpha}}\right)+\int_{1}^{x_{0}} d x \log \left(1+\frac{C n^{p}}{x^{\alpha}}\right) \\
& +\int_{x_{0}}^{n} d x \log \left(1+\frac{C n^{p}}{x^{\alpha}}\right) \\
\leq & \int_{0}^{1} d x \log \left(\frac{1+C n^{p}}{x^{\alpha}}\right)+\int_{1}^{x_{0}} d x \log \left(1+C n^{p}\right) \\
& +\int_{x_{0}}^{n} d x\left(\frac{C n^{p}}{x^{\alpha}}\right) \\
= & \log \left(1+C n^{p}\right)+\alpha \int_{0}^{1} d x \log \left(\frac{1}{x}\right) \\
& +\left(x_{0}-1\right) \log \left(1+C n^{p}\right)+\frac{C n^{p}}{\alpha-1}\left(\frac{1}{x_{0}^{\alpha-1}}-\frac{1}{n^{\alpha-1}}\right) .
\end{array}
$$

Replacing $x_{0}$ by its value and checking separately the two cases $\frac{p}{\alpha}<1$ and $\frac{p}{\alpha} \geq 1$ leads then to the conclusion.

This lemma allows us to deduce the following.

Proposition 2.3: There exists a constant $K>0$ (possibly depending on $\alpha$ ) such that for all sufficiently large $n$

$$
\mathbb{E}\left(D_{n}^{0}\right) \leq K n^{\frac{d-1}{d}+\left(\frac{1}{\alpha} \wedge \frac{1}{d}\right)} \log n
$$

so $\mathbb{E}\left(D_{n}^{0}\right)$ is sublinear in $n$ if $\alpha>d$.

Proof: Set $m=n^{\frac{1}{d}}$ (which need not be an integer). First note that

$$
\begin{aligned}
\mathbb{E}\left(D_{n}^{0}\right) & =n \mathbb{E}\left(\log \left(1+\sqrt{P n} g\left(2\left|x_{1}^{(1)}\right|\right)\right)\right) \\
& =m^{d-1} \int_{0}^{m} d x \log \left(1+\frac{\sqrt{P} m^{d / 2}}{(2 x)^{\alpha / 2}}\right) .
\end{aligned}
$$

So we obtain by Lemma 2.2 that for sufficiently large $n$

$$
\mathbb{E}\left(D_{n}^{0}\right) \leq K m^{d-1} m^{\left(\frac{d}{\alpha} \wedge 1\right)} \log m=\frac{K}{d} n^{\frac{d-1}{d}+\left(\frac{1}{\alpha} \wedge \frac{1}{d}\right)} \log n,
$$

and this concludes the proof.

There remains to prove that the sublinear behavior of $D_{n}^{0}$ in $n$ takes place almost surely. We prove this by showing that the 
deviation of $D_{n}^{0}$ from its average is indeed almost surely sublinear in $n$.

Proposition 2.4: Fix $\alpha>0$. Then for any $\varepsilon>0$, we have

$$
\lim _{n \rightarrow \infty} \frac{\left|D_{n}^{0}-\mathbb{E}\left(D_{n}^{0}\right)\right|}{n^{\frac{1}{2}+\varepsilon}}=0, \text { almost surely. }
$$

Proof: What we are going to use here is Hoeffding's inequality (see [7]). We first note that $D_{n}^{0}$ is the sum of $n$ independent random variables

$$
d_{k}=\log \left(1+\sqrt{n P} g\left(2\left|x_{k}^{(1)}\right|\right)\right) .
$$

However, each of these random variables is unbounded, since $\left|x_{k}^{(1)}\right|$ can be arbitrarily close to zero. We are going to show that with a certain scaling factor, they are all bounded away from zero with high probability as $n$ goes to infinity, and that under the condition that they are effectively bounded away from zero, $D_{n}^{0}$ concentrates around its mean with a deviation of order less than $n^{\frac{1}{2}+\varepsilon}$ for any $\varepsilon>0$. Let us then fix $\eta>0$ and compute the probability that any of the $\left|x_{k}^{(1)}\right|$ is smaller than $n^{-\frac{d-1}{d}-\eta}$. Denoting by $x_{\min }$ the vector $x_{k}$ whose first component is minimal (in absolute value), we obtain by the union bound that

$$
\begin{aligned}
\mathbb{P}\left(\left|x_{\min }^{(1)}\right|<n^{-\frac{d-1}{d}-\eta}\right) & \leq n \mathbb{P}\left(\left|x_{1}^{(1)}\right|<n^{-\frac{d-1}{d}-\eta}\right) \\
& =n \frac{n^{-\frac{d-1}{d}-\eta}}{n^{\frac{1}{d}}}=n^{-\eta}
\end{aligned}
$$

and this probability is arbitrarily small for any $\eta>0$. On the other hand, under the condition that

$$
\left|x_{\min }^{(1)}\right| \geq n^{-\frac{d-1}{d}-\eta}
$$

the $\left|x_{k}^{(1)}\right|$ remain independent and identically distributed (i.i.d.) random variables, as the following calculation shows. Let $t_{1}, \ldots, t_{n} \in\left[n^{-\frac{d-1}{d}-\eta}, n\right]$; we then have

$$
\begin{aligned}
& \mathbb{P}\left(\left|x_{1}^{(1)}\right| \geq t_{1}, \ldots,\left|x_{n}^{(1)}\right| \geq t_{n}|| x_{\min }^{(1)} \mid \geq n^{-\frac{d-1}{d}-\eta}\right) \\
& =\frac{\mathbb{P}\left(\left|x_{1}^{(1)}\right| \geq t_{1}, \ldots,\left|x_{n}^{(1)}\right| \geq t_{n}\right)}{\mathbb{P}\left(\left|x_{1}^{(1)}\right| \geq n^{-\frac{d-1}{d}-\eta}, \ldots,\left|x_{n}^{(1)}\right| \geq n^{-\frac{d-1}{d}-\eta}\right)} \\
& =\prod_{k=1}^{n} \frac{\mathbb{P}\left(\left|x_{k}^{(1)}\right| \geq t_{k}\right)}{\mathbb{P}\left(\left|x_{k}^{(1)}\right| \geq n^{-\frac{d-1}{d}-\eta}\right)} \\
& =\prod_{k=1}^{n} \mathbb{P}\left(\left|x_{k}^{(1)}\right| \geq t_{k}|| x_{k}^{(1)} \mid \geq n^{-\frac{d-1}{d}-\eta}\right) \\
& =\prod_{k=1}^{n} \mathbb{P}\left(\left|x_{k}^{(1)}\right| \geq t_{k}|| x_{\min }^{(1)} \mid \geq n^{-\frac{d-1}{d}-\eta}\right)
\end{aligned}
$$

since the $x_{k}^{(1)}$ are independent. Moreover, under the condition that $\left|x_{\min }^{(1)}\right| \geq n^{-\frac{d-1}{d}-\eta}$, the random variables $d_{k}$ are bounded

$$
\begin{aligned}
\left|d_{k}\right| & \leq \log \left(1+\sqrt{n P} g\left(2 n^{-\frac{d-1}{d}-\eta}\right)\right) \\
& \leq \log \left(1+\sqrt{P} n^{\frac{1}{2}+\frac{\alpha}{2}\left(\frac{d-1}{d}+\eta\right)}\right) \\
& \leq C_{1}(\eta)+C_{2}(\eta) \log n .
\end{aligned}
$$

We can, therefore, apply Hoeffding's inequality [7] which states that for all $\lambda>0$ and sufficiently large $n$

$$
\begin{aligned}
\mathbb{P}\left(\left|D_{n}^{0}-\mathbb{E}\left(D_{n}^{0}\right)\right| \geq\right. & \left.\lambda \sqrt{n}|| x_{\min }^{(1)} \mid \geq n^{-\frac{d-1}{d}-\eta}\right) \\
& \leq 2 \exp \left(-\frac{2 \lambda^{2}}{\left(C_{1}(\eta)+C_{2}(\eta) \log n\right)^{2}}\right)
\end{aligned}
$$

and replacing $\lambda$ by $\lambda n^{\varepsilon}$ in the preceding inequality gives

$$
\begin{aligned}
\mathbb{P}\left(\left|D_{n}^{0}-\mathbb{E}\left(D_{n}^{0}\right)\right| \geq\right. & \left.\lambda n^{\frac{1}{2}+\varepsilon}|| x_{\min }^{(1)} \mid \geq n^{-\frac{d-1}{d}-\eta}\right) \\
& \leq 2 \exp \left(-\frac{2 \lambda^{2} n^{2 \varepsilon}}{\left(C_{1}(\eta)+C_{2}(\eta) \log n\right)^{2}}\right) .
\end{aligned}
$$

In conclusion, we have

$$
\begin{aligned}
\mathbb{P} & \left(\left|D_{n}^{0}-\mathbb{E}\left(D_{n}^{0}\right)\right| \geq \lambda n^{\frac{1}{2}+\varepsilon}\right) \\
\leq & \mathbb{P}\left(\left|x_{\min }^{(1)}\right|<n^{-\frac{d-1}{d}-\eta}\right) \\
& +\mathbb{P}\left(\left|D_{n}^{0}-\mathbb{E}\left(D_{n}^{0}\right)\right| \geq \lambda n^{\frac{1}{2}+\varepsilon}|| x_{\min }^{(1)} \mid \geq n^{-\frac{d-1}{d}-\eta}\right) \\
& \leq n^{-\eta}+2 \exp \left(-\frac{2 \lambda^{2} n^{2 \varepsilon}}{\left(C_{1}(\eta)+C_{2}(\eta) \log n\right)^{2}}\right) .
\end{aligned}
$$

Choosing $\eta>1$, we therefore obtain that

$$
\sum_{n \geq 1} \mathbb{P}\left(\left|D_{n}^{0}-\mathbb{E}\left(D_{n}^{0}\right)\right| \geq \lambda n^{\frac{1}{2}+\varepsilon}\right)<\infty
$$

so by the Borel-Cantelli lemma, we have for any $\varepsilon>0$ and $\lambda>0$

$$
\mathbb{P}\left(\frac{\left|D_{n}^{0}-\mathbb{E}\left(D_{n}^{0}\right)\right|}{n^{\frac{1}{2}+\varepsilon}} \geq \lambda \text { infinitely often }\right)=0
$$

which implies the result.

We summarize the results obtained so far in the following theorem.

Theorem 2.5: If $\alpha>d \vee 2(d-2)$ (that is, $\alpha>d$ when $d \leq 4$ ) and there is no absorption (that is, $\beta=0$ ), then the maximum achievable rate $R$ per communication pair in a large uniformly distributed network decreases almost surely to zero as the number of users gets large. More precisely, under the above assumption, there exist a constant $K>0$ such that for sufficiently large $n$

$$
R \leq K\left(\frac{\log n}{n^{\frac{1}{d}-\frac{1}{\alpha}}}+\frac{1}{\sqrt{n}}\right) \quad \text { almost surely. }
$$

Note that the last estimate comes from the fact that

$$
R \leq \frac{D_{n}^{0}}{n / 8+o(n)}
$$

and Propositions 2.3 and 2.4.

Remark 2.6: As a by-product, the preceding analysis also gives an upper bound on the maximal amount of information that can be carried from one part of the network to the other. This amount is bounded above by $C_{n}$, which in turn is bounded above by $K\left(n^{\frac{d-1}{d}+\frac{1}{\alpha}} \log n+\sqrt{n}\right)$, almost surely for sufficiently large $n$. 


\section{B. Case With Absorption}

In the following, we assume that $\beta>0$. Starting from (3), we follow the lines of the preceding subsection: using the fact that $P_{k} \leq n P$, we have

$$
C_{n} \leq \sum_{k=1}^{n} \log \left(1+n P \lambda_{k}^{2}\right)
$$

Now, since $\lambda_{k}^{2}$ are the eigenvalues of the matrix $G G^{\dagger}$, repeating the majorization argument of Section II-A gives

$$
C_{n} \leq \sum_{k=1}^{n} \log \left(1+n P\left(G G^{\dagger}\right)_{k k}\right)
$$

Moreover, since $g$ is decreasing, we have

$$
\left(G G^{\dagger}\right)_{k k}=\sum_{l=1}^{n}\left|G_{k l}\right|^{2}=\sum_{l=1}^{n} g\left(\left|x_{k}-y_{l}\right|\right)^{2} \leq n g\left(\left|x_{k}^{(1)}\right|\right)^{2} \text {. }
$$

So we finally obtain

$$
C_{n} \leq D_{n}:=\sum_{k=1}^{n} \log \left(1+P n^{2} g\left(\left|x_{k}^{(1)}\right|\right)^{2}\right) .
$$

We need now the following technical lemma, similar to Lemma 2.2.

Lemma 2.7: For any $C, p, \alpha, \beta>0$, there exists a constant $K>0$ such that for all sufficiently large $n$, we have

$$
\int_{0}^{n} d x \log \left(1+\frac{C n^{p} e^{-\beta x}}{x^{\alpha}}\right) \leq K(\log n)^{2} .
$$

Proof: The proof follows the lines of that of Lemma 2.2; set $x_{0}=\frac{p}{\beta} \log n$ (which is smaller than $n$ for sufficiently large $n$ ) and compute

$$
\begin{array}{rl}
\int_{0}^{n} & d x \log \left(1+\frac{C n^{p} e^{-\beta x}}{x^{\alpha}}\right) \\
\leq & \int_{0}^{1} d x \log \left(1+\frac{C n^{p} e^{-\beta x}}{x^{\alpha}}\right) \\
& +\int_{1}^{n} d x \log \left(1+C n^{p} e^{-\beta x}\right) \\
\leq & \int_{0}^{1} d x \log \left(\frac{1+C n^{p}}{x^{\alpha}}\right) \\
& +\int_{1}^{x_{0}} d x \log \left(1+C n^{p}\right)+\int_{x_{0}}^{n} d x C n^{p} e^{-\beta x} \\
= & \log \left(1+C n^{p}\right)+\alpha \int_{0}^{1} d x \log \left(\frac{1}{x}\right) \\
& +\left(x_{0}-1\right) \log \left(1+C n^{p}\right)+\frac{C n^{p}}{\beta}\left(e^{-\beta x_{0}}-e^{-\beta n}\right) .
\end{array}
$$

Replacing $x_{0}$ by its value then proves the lemma.

From this, we deduce the following upper bound on the average behavior of $D_{n}$.

Proposition 2.8: There exists a constant $K>0$ (possibly depending on $\alpha$ or $\beta$ ) such that for all sufficiently large $n$

$$
\mathbb{E}\left(D_{n}\right) \leq K n^{\frac{d-1}{d}}(\log n)^{2}
$$

so $\mathbb{E}\left(D_{n}\right)$ is sublinear in $n$.
Proof: Set $m=n^{\frac{1}{d}}$. First note that

$$
\begin{aligned}
\mathbb{E}\left(D_{n}\right) & =n \mathbb{E}\left(\log \left(1+P n^{2} g\left(\left|x_{1}^{(1)}\right|\right)^{2}\right)\right) \\
& =m^{d-1} \int_{0}^{m} d x \log \left(1+\frac{P m^{2 d} e^{-\beta x}}{x^{\alpha}}\right) .
\end{aligned}
$$

So we obtain by Lemma 2.7 that for sufficiently large $n$

$$
\mathbb{E}\left(D_{n}\right) \leq K m^{d-1}(\log m)^{2}=\frac{K}{d^{2}} n^{\frac{d-1}{d}}(\log n)^{2}
$$

and this concludes the proof.

As before, there remains to prove that the sublinear behavior of $D_{n}$ in $n$ takes place almost surely. We prove this using the following concentration result.

Proposition 2.9: Fix $\alpha>0, \beta>0$. Then for any $\varepsilon>0$, we have

$$
\lim _{n \rightarrow \infty} \frac{\left|D_{n}-\mathbb{E}\left(D_{n}\right)\right|}{n^{\frac{1}{2}+\varepsilon}}=0, \text { almost surely. }
$$

Proof: The proof is identical to that of Proposition 2.4, so we do not repeat it here.

We summarize the results obtained so far in the following theorem.

Theorem 2.10: If there is absorption (that is, $\beta>0$ ), then the maximum achievable rate $R$ per communication pair in a large uniformly distributed network decreases almost surely to zero as the number of users gets large. More precisely, under the above assumption, there exists a constant $K>0$ such that for sufficiently large $n$

$$
R \leq K\left(\frac{(\log n)^{2}}{n^{\frac{1}{d}}}+\frac{1}{\sqrt{n}}\right) \quad \text { almost surely. }
$$

Note that the last estimate comes from the fact that

$$
R \leq \frac{D_{n}}{n / 8+o(n)}
$$

and Propositions 2.8 and 2.9.

\section{REGULAR NETWORKS}

Let us now consider the case where the network is a regular network in the sense that the users are placed on a regular grid inside $\Omega_{n}$. For simplicity, we will assume that there are $n$ users on each side and that $n=m^{d}$ for some integer $m$. The positions of the users on the left- and the right-hand side of the region $\Omega_{n}$ are therefore given by

$$
x_{k}=\left(-k_{1}+0.5, k_{2}-0.5, \ldots, k_{d}-0.5\right)
$$

and

$$
y_{l}=\left(l_{1}-0.5, l_{2}-0.5, \ldots, l_{d}-0.5\right)
$$

where $k=\left(k_{1}, \ldots, k_{d}\right)$ and $l=\left(l_{1}, \ldots, l_{d}\right)$ denote from now on multiple indices ranging from $(1, \ldots, 1)$ to $(m, \ldots, m)$. For notational simplicity, we will go on writing $k=1, \ldots, n$ for an enumeration of all the multiple indices. 
We consider that these $2 n$ users wish to form $n$ communication pairs, choosing their correspondent at random. An argument similar to that developed in the previous section shows that there will be about $n / 4$ communications needing to cross the imaginary boundary $x^{(1)}=0$ from left to right. Repeating then the argument of the previous section leads to the following upper bound on the maximum achievable rate per communication pair in the network:

where

$$
R \leq \frac{C_{n}}{n / 4+o(n)}
$$

$$
C_{n}:=\max _{P_{k} \geq 0: \sum_{k=1}^{n}} \sum_{P_{k} \leq n P}^{n} \log \left(1+P_{k} \lambda_{k}^{2}\right) .
$$

Here, $\lambda_{k}$ are the singular values of the matrix $G$, whose entries $G_{i j}=g\left(\left|x_{i}-y_{j}\right|\right)$ are determinisitic in the present context.

\section{A. No Absorption Case}

Let us assume that $\beta=0$. We will show in the following a better result than that of Section II-A, in the sense that we do not need any more the assumption that $\alpha>2(d-2)$, but this requires us to be a little more careful in the majoration procedure. Let us first note that for any fixed vector $\left(P_{1}, \ldots, P_{n}\right)$, we have

$$
\sum_{k=1}^{n} \log \left(1+P_{k} \lambda_{k}^{2}\right) \leq \sum_{k=1}^{n} \log \left(1+P_{k}\left(G G^{\dagger}\right)_{k k}\right)
$$

by the same majorization argument as that of Section II-B. Moreover, since $g$ is decreasing, we have

$$
\begin{aligned}
\left(G G^{\dagger}\right)_{k k} & =\sum_{l=1}^{n} g\left(\left|x_{k}-y_{l}\right|\right)^{2} \leq \sum_{l=1}^{n} g\left(\left|x_{k}^{(1)}-y_{l}^{(1)}\right|\right)^{2} \\
& =m^{d-1} \sum_{l_{1}=1}^{m} g\left(k_{1}+l_{1}-1\right)^{2}
\end{aligned}
$$

(recall that $x_{k}^{(1)}=-k_{1}+0.5, y_{l}^{(1)}=l_{1}-0.5$, and $n=m^{d}$ where $m$ is an integer). Let us compute

$$
\begin{aligned}
\sum_{l_{1}=1}^{m} g\left(k_{1}+l_{1}-1\right)^{2} & =\sum_{l_{1}=1}^{m} \frac{1}{\left(k_{1}+l_{1}-1\right)^{\alpha}} \\
& \leq \frac{1}{k_{1}^{\alpha}}+\int_{1}^{m} d y \frac{1}{\left(k_{1}+y-1\right)^{\alpha}} \\
& \leq \frac{1}{k_{1}^{\alpha}}+\frac{1}{\alpha-1} \frac{1}{k_{1}^{\alpha-1}} \leq \frac{\alpha}{\alpha-1} \frac{1}{k_{1}^{\alpha-1}} .
\end{aligned}
$$

This leads to the following upper bound:

$$
C_{n} \leq \max _{P_{k} \geq 0: \sum_{k=1}^{n} P_{k} \leq n P} \sum_{k=1}^{n} \log \left(1+\frac{\alpha}{\alpha-1} \frac{P_{k} m^{d-1}}{k_{1}^{\alpha-1}}\right)
$$

which can be rewritten as

$$
C_{n} \leq \operatorname{Pax}_{\tilde{P}_{k} \geq 0: \sum_{k=1}^{n} \tilde{P}_{k} \leq \frac{\alpha}{\alpha-1} n P} \sum_{k=1}^{n} \log \left(1+\frac{\tilde{P}_{k} m^{d-1}}{k_{1}^{\alpha-1}}\right) .
$$

This maximization problem has the well-known "water-filling" solution

$$
C_{n}=\sum_{k=1}^{n} \log \left(\frac{\nu m^{d-1}}{k_{1}^{\alpha-1}}\right)^{+}
$$

where $\nu$ satisfies the constraint

$$
\sum_{k=1}^{n}\left(\nu-\frac{k_{1}^{\alpha-1}}{m^{d-1}}\right)^{+}=\frac{\alpha}{\alpha-1} n P
$$

and $a^{+}$denotes the positive part of $a \in \mathbb{R}$. We need now the following two technical lemmas.

Lemma 3.1: Let $\alpha>d$ and $\nu$ satisfy (8). There exists then a constant $K>0$ (possibly depending on $\alpha$ ) such that for all sufficiently large $n$, we have

$$
\nu \leq K n^{\frac{1}{d}\left(1-\frac{d}{\alpha}\right)} .
$$

Proof: Equation (8) implies that

$$
\begin{aligned}
\frac{\alpha}{\alpha-1} m P & =\sum_{k_{1}=1}^{m}\left(\nu-\frac{k_{1}^{\alpha-1}}{m^{d-1}}\right)^{+} \\
& \geq \int_{1}^{m} d x\left(\nu-\frac{x^{\alpha-1}}{m^{d-1}}\right)^{+} \\
& =\int_{1}^{x_{0}} d x\left(\nu-\frac{x^{\alpha-1}}{m^{d-1}}\right)
\end{aligned}
$$

where $x_{0}=\left(\nu m^{d-1}\right)^{\frac{1}{\alpha-1}}$. Computing this last expression gives

$$
\begin{aligned}
\frac{\alpha}{\alpha-1} m P & \geq \nu\left(x_{0}-1\right)-\frac{x_{0}^{\alpha}-1}{\alpha m^{d-1}} \\
& \geq \nu^{\frac{\alpha}{\alpha-1}} m^{\frac{d-1}{\alpha-1}} \frac{\alpha-1}{\alpha}-\nu:=F(\nu) .
\end{aligned}
$$

Since $F$ is increasing on the domain where it is positive, we then obtain that $\nu \leq \nu_{0}$, where $\nu_{0}$ satisfies the equation

$$
F\left(\nu_{0}\right)=\frac{\alpha}{\alpha-1} m P .
$$

This equation in turn implies that

so

$$
\nu_{0}^{\frac{\alpha}{\alpha-1}} m^{\frac{d-1}{\alpha-1}} \frac{\alpha-1}{\alpha} \geq \frac{\alpha}{\alpha-1} m P
$$

$$
\nu_{0} \geq\left(P\left(\frac{\alpha}{\alpha-1}\right)^{2}\right)^{\frac{\alpha-1}{\alpha}} m^{\left(1-\frac{d-1}{\alpha-1}\right) \frac{\alpha-1}{\alpha}}:=K_{\alpha} m^{1-\frac{d}{\alpha}}
$$

and

$$
\begin{aligned}
\nu_{0}^{-\frac{1}{\alpha-1}} m^{-\frac{d-1}{\alpha-1}} & \leq K_{\alpha}^{-\frac{1}{\alpha-1}} m^{-\frac{1}{\alpha-1}\left(1-\frac{d}{\alpha}\right)-\frac{d-1}{\alpha-1}} \\
& =K_{\alpha}^{-\frac{1}{\alpha-1}} m^{-\frac{d}{\alpha}} \underset{m \rightarrow \infty}{\longrightarrow} 0
\end{aligned}
$$

since $\alpha>d$. Now, since

$$
F\left(\nu_{0}\right)=\nu_{0}^{\frac{\alpha}{\alpha-1}} m^{\frac{d-1}{\alpha-1}}\left(\frac{\alpha-1}{\alpha}-\nu_{0}^{-\frac{1}{\alpha-1}} m^{-\frac{d-1}{\alpha-1}}\right)
$$

we obtain that for sufficently large $m$, there exists $K>0$ (depending on $\alpha$ ) such that

$$
\frac{\alpha}{\alpha-1} m P=F\left(\nu_{0}\right) \geq K \nu_{0}^{\frac{\alpha}{\alpha-1}} m^{\frac{d-1}{\alpha-1}} .
$$

This implies finally that

$$
\nu \leq \nu_{0} \leq K m^{\left(1-\frac{d-1}{\alpha-1}\right) \frac{\alpha-1}{\alpha}}=K m^{1-\frac{d}{\alpha}}
$$

which concludes the proof.

Lemma 3.2: For any $k_{0} \geq 1$, we have

$$
\sum_{k_{1}=1}^{k_{0}} \log \left(\frac{k_{0}}{k_{1}}\right) \leq k_{0} \text {. }
$$


Proof: Let us simply compute

$$
\begin{aligned}
\sum_{k_{1}=1}^{k_{0}} \log \left(\frac{k_{0}}{k_{1}}\right) & =k_{0} \log k_{0}-\sum_{k_{1}=1}^{k_{0}} \log k_{1} \\
& \leq k_{0} \log k_{0}-\int_{1}^{k_{0}} d x \log x \\
& =k_{0} \log k_{0}-k_{0} \log k_{0}+k_{0}-1 \leq k_{0}
\end{aligned}
$$

This allows us to establish the following proposition.

Proposition 3.3: Let $\alpha>d$. There exists then a constant $K>0$ (possibly depending on $\alpha$ ) such that for all sufficiently large $n$, we have

$$
C_{n} \leq K n^{\frac{d-1}{d}+\frac{1}{\alpha}}
$$

So $C_{n}$ is sublinear in $n$ when $\alpha>d$.

Proof: From the preceding analysis, we have the following upper bound on $C_{n}$ :

$$
C_{n} \leq m^{d-1} \sum_{k_{1}=1}^{m} \log \left(\frac{\nu m^{d-1}}{k_{1}^{\alpha-1}}\right)^{+}
$$

where $\nu$ satisfies the constraint (8). Let $k_{0}$ denote the smallest integer such that

$$
\left(\nu m^{d-1}\right)^{\frac{1}{\alpha-1}} \leq k_{0}
$$

We then obtain

$$
C_{n} \leq m^{d-1} \sum_{k=1}^{k_{0}} \log \left(\frac{k_{0}^{\alpha-1}}{k_{1}^{\alpha-1}}\right) \leq m^{d-1}(\alpha-1) k_{0}
$$

by Lemma 3.2. On the other hand, by Lemma 3.1, there exists $K>0$ such that

$$
k_{0} \leq K m^{\left(1-\frac{d}{\alpha}+d-1\right) \frac{1}{\alpha-1}}+1=K m^{\frac{d}{\alpha}}+1
$$

for sufficiently large $m$, which in turn implies

$$
C_{n} \leq K m^{d-1+\frac{d}{\alpha}}
$$

and this completes the proof.

This proposition leads directly to the following theorem.

Theorem 3.4: If $\alpha>d$ and there is no absorption (that is, $\beta=0$ ), then the maximum achievable rate $R$ per communication pair in a large regular network decreases almost surely to zero as the number of users gets large.

We obtain here the same result as the one obtained for uniformly distributed networks, without assuming that the matrix $G$ is nonnegative definite, that is, without the assumption that $\alpha>2(d-2)$.

\section{B. Case With Absorption}

In the case where there is absorption (that is, $\beta>0$ ), we follow the lines of Section II-B and obtain easily

$$
C_{n} \leq \sum_{k=1}^{n} \log \left(1+n P \lambda_{k}^{2}\right) \leq \sum_{k=1}^{n} \log \left(1+n P\left(G G^{\dagger}\right)_{k k}\right)
$$

$$
\begin{aligned}
& \leq m^{d-1} \sum_{k_{1}=1}^{m} \log \left(1+P m^{2 d} g\left(k_{1}\right)^{2}\right) \\
& \leq m^{d-1} \sum_{k_{1}=1}^{m} \log \left(1+P m^{2 d} e^{-\beta k_{1}}\right) \\
& \leq m^{d-1} \int_{0}^{m} d x \log \left(1+P m^{2 d} e^{-\beta x}\right) \\
& \leq \frac{K}{d^{2}} n^{\frac{d-1}{d}}(\log n)^{2}
\end{aligned}
$$

by Lemma 2.7, so this proves the following theorem.

Theorem 3.5: If there is absorption (that is, $\beta>0$ ), then the maximum achievable rate $R$ per communication pair in a large regular network decreases almost surely to zero as the number of users gets large.

\section{Conclusion AND Perspectives}

We have proved that under minimal assumptions (that is, with a power decay of order $1 / r^{\alpha}$ with $\alpha>d \vee 2(d-2)$ or in the presence of absorption), the maximum achievable rate per communication pair in a large extended ad hoc network has to decrease to zero as the number of users gets large. However, we have seen that our scaling law is not as tight as the one obtained in [1], [2]. In order to get a better result, a precise study of the behavior of the singular values $\lambda_{k}$ is necessary.

\section{APPENDIX}

\section{The Matrix $G$ Is Nonnegative Definite IN THE NO-ABSORPTION CASE}

Let us first consider the one-dimensional case (with $\alpha>0$ ). In this case, since $y_{j}=-x_{j}$, the entries of the matrix $G$ are given by

$$
\begin{aligned}
G_{i j} & =g\left(\left|x_{i}-y_{j}\right|\right)=g\left(x_{i}+x_{j}\right)=\frac{1}{\left(x_{i}+x_{j}\right)^{\alpha / 2}} \\
& =\int_{0}^{\infty} d t \frac{t^{\alpha / 2-1}}{\Gamma(\alpha / 2)} e^{-\left(x_{i}+x_{j}\right) t}
\end{aligned}
$$

where $\Gamma$ is the Euler Gamma function. This implies that $G$ is nonnegative definite, since

$$
\sum_{i, j=1}^{n} G_{i j} c_{i} c_{j}=\int_{0}^{\infty} d t \frac{t^{\alpha / 2-1}}{\Gamma(\alpha / 2)}\left(\sum_{i=1}^{n} e^{-x_{i} t} c_{i}\right)^{2} \geq 0 .
$$

Let us now consider the higher dimensional case together with the assumption that $\alpha>2(d-2)$. We have the expression for the entries of the matrix $G$

$$
G_{i j}=\frac{1}{\left(\left(x_{i}^{(1)}+x_{j}^{(1)}\right)^{2}+\sum_{k=2}^{d}\left|x_{i}^{(k)}-x_{j}^{(k)}\right|^{2}\right)^{\alpha / 4}}
$$

so using the fact that the Fourier transform of

$$
f_{a}\left(x_{2}, \ldots, x_{d}\right)=\frac{1}{\left(a^{2}+\left|x_{2}\right|^{2}+\cdots+\left|x_{d}\right|^{2}\right)^{\alpha / 4}}
$$


is given by (see [8, Formulas I.2.7 and I.18.29])

$$
\begin{aligned}
& \hat{f}_{a}\left(\xi_{2}, \ldots, \xi_{d}\right)=C_{\alpha, d}\left(\frac{\sqrt{\left|\xi_{2}\right|^{2}+\cdots+\left|\xi_{d}\right|^{2}}}{a}\right)^{\frac{\alpha}{4}-\frac{d-1}{2}} \\
& \cdot K_{\frac{\alpha}{4}-\frac{d-1}{2}}\left(a \sqrt{\left|\xi_{2}\right|^{2}+\cdots+\left|\xi_{d}\right|^{2}}\right)
\end{aligned}
$$

where $K_{\nu}$ is the modified Bessel function of second kind and of order $\nu$, we obtain that

$$
\begin{aligned}
G_{i j}= & \frac{C_{\alpha, d}}{(2 \pi)^{d-1}} \int_{\mathbb{R}^{d-1}} d \xi_{2} \cdots d \xi_{d}\left(\frac{\sqrt{\left|\xi_{2}\right|^{2}+\cdots+\left|\xi_{d}\right|^{2}}}{x_{i}^{(1)}+x_{j}^{(1)}}\right)^{\frac{\alpha}{4}-\frac{d-1}{2}} \\
& \cdot K_{\frac{\alpha}{4}-\frac{d-1}{2}}\left(\left(x_{i}^{(1)}+x_{j}^{(1)}\right) \sqrt{\left|\xi_{2}\right|^{2}+\cdots+\left|\xi_{d}\right|^{2}}\right) \\
& \cdot e^{i \xi_{2}\left(x_{i}^{(2)}-x_{j}^{(2)}\right)+\cdots+i \xi_{d}\left(x_{i}^{(d)}-x_{j}^{(d)}\right)} .
\end{aligned}
$$

Since by [9, Formula 9.6.23], we have

$$
\frac{1}{r^{\nu}} K_{\nu}(r)=\frac{\sqrt{\pi}}{2^{\nu} \Gamma\left(\nu+\frac{1}{2}\right)} \int_{0}^{\infty} d t e^{-r \cosh (t)} \sinh ^{2 \nu}(t)
$$

for $\nu>-\frac{1}{2}$, we obtain that the matrix $M$ whose entries are given by

$$
\begin{aligned}
M_{i j}= & \left(\frac{\sqrt{\left|\xi_{2}\right|^{2}+\cdots+\left|\xi_{d}\right|^{2}}}{x_{i}^{(1)}+x_{j}^{(1)}}\right)^{\frac{\alpha}{4}-\frac{d-1}{2}} \\
& \quad \cdot K_{\frac{\alpha}{4}-\frac{d-1}{2}}\left(\left(x_{i}^{(1)}+x_{j}^{(1)}\right) \sqrt{\left|\xi_{2}\right|^{2}+\cdots+\left|\xi_{d}\right|^{2}}\right)
\end{aligned}
$$

is nonnegative definite if

$$
\frac{\alpha}{4}-\frac{d-1}{2}>-\frac{1}{2}, \quad \text { that is, } \alpha>2(d-2) .
$$

So under the same assumption, $G$ is a convex combination of products of nonnegative definite matrices, it is therefore itself nonnegative definite.

\section{ACKNOWLEDGMENT}

The authors would like to thank Shashibhushan Borade, Michael Gastpar, Piyush Gupta, Shan-Yuan Ho, P. R. Kumar, David N. C. Tse, Rüdiger Urbanke, and Feng Xue for many stimulating discussions.

\section{REFERENCES}

[1] P. Gupta and P. R. Kumar, "The capacity of wireless networks," IEEE Trans. Inf. Theory, vol. 46, no. 2, pp. 388-404, Mar. 2000.

[2] P. R. Kumar and L.-L. Xie, "A network information theory for wireless communications: Scaling laws and optimal operation," IEEE Trans. Inf. Theory, vol. 50, no. 5, pp. 748-767, May 2004.

[3] P. Gupta and P. R. Kumar, "Internets in the sky: The capacity of three dimensional wireless networks," Commun. Inf. Syst., vol. 1, no. 1, pp. 33-50, 2001.

[4] T. M. Cover and J. A. Thomas, Elements of Information Theory. New York: Wiley, 1991.

[5] E. Telatar, "Capacity of multi-antenna Gaussian channels," Europ. Trans. Telecommun., vol. 10, no. 6, pp. 585-595, 1999.

[6] A. W. Marshall and I. Olkin, Inequalities: Theory of Majorization and its Applications, ser. Mathematics in Science and Engineering. New York-London: Academic, 1979, vol. 143.

[7] W. Hoeffding, "Probability inequalities for sums of bounded random variables," J. Amer. Statist. Assoc., vol. 58, pp. 13-30, 1963.

[8] F. Oberhettinger, Tables of Fourier Transforms and Fourier Transforms of Distributions. Berlin, Germany: Springer-Verlag, 1990.

[9] M. Abramowitz and I. A. Stegun, Handbook of Mathematical Functions. Washington, DC: Nat. Bur. Stds., 1966. 